Methods Prospective case series.

Results 51 patients admitted for stroke workup were recruited across 2 major tertiary centre's in Sydney to compare WT monitoring for 2 days versus S-patch monitoring for 4 days in the detection of AF. The efficacy to detect AF using both technologies across 76 hours of telemetry was assessed via data extractions and Cardiologist review. A matrix was used to measure nursing/patient satisfaction and setup/resource times were assessed.

$84-94 \%$ of patients and $75-95 \%$ of nursing preferred the $\mathrm{S}$-Patch. Non-parametric tests indicate significant time saving for removal of S-Patch versus WT [2.2 mins vs 5.1 mins $(p=0.00)]$. Efficacy of S-Patch to detect AF following Cardiologist review was greater than WT, with 7 patients identified with AF by S-Patch versus 1 using WT. The S-patch had a false positive rate of $78 \%$.

Conclusion The S-patch had a higher detection rate of AF compared to WT. This allows patients to be anticoagulated appropriately for the prevention of further stroke. Analysis shows patients and staff overwhelmingly prefer the S-Patch. The S-Patch is sensitive in the detection of AF however it showed a high false positive rate. We are confident that further refinement and advances will provide a novel device in the detection of AF.

\section{SEPARATING STROKE FROM VESTIBULAR NEURITIS BY VESTIBULAR FUNCTION TEST PARAMETERS}

2,3,4,1 Zeljka Calic* ${ }^{5}$ Benjamin Nham, ${ }^{5}$ Rachel Taylor, ${ }^{5}$ Allison Young, ${ }^{4}$ Craig Anderson, ${ }^{2,3,1}$ Cecilia Cappelen-Smith, ${ }^{2,3,1}$ Dennis Cordato, ${ }^{5}$ Miriam Welgampola. ${ }^{1}$ Department of Neurophysiology, Liverpool Hospital, Liverpool, NSW, Australia; ${ }^{2}$ South Western Sydney Clinical School, UNSW, Sydney, NSW, Australia; 3 Ingham Institute for Applied Medical Research, Liverpool, NSW, Australia; ${ }^{4}$ The George Institute for Global Heath, University of New South Wales, Sydney, NSW, Australia; ${ }^{5}$ Institute of Clinical Neurosciences, Royal Prince Alfred Hospital, Central Clinical School University of Sydney, Sydney, NSW, Australia

\subsection{6/jnnp-2019-anzan.72}

Introduction Vestibular neuritis (VN) and posterior circulation stroke (PCS) are the commonest causes of acute vestibular syndrome (AVS). We aim to identify discriminators of $\mathrm{VN}$ from PCS by testing all five vestibular end-organs in patients presenting with AVS.

Methods Three-dimensional video-head impulse test (v-HIT), cervical and ocular-vestibular evoked myogenic potentials (cand oVEMP) and subjective visual horizontal (SVH) tests were performed in 22 patients with $\mathrm{VN}$ and 22 with PCS. Ipsilesional horizontal, anterior and posterior canal (HC, AC, PC) v-HIT gain and first catch-up saccade characteristics, VEMP amplitude asymmetry-ratios were compared.

Results All VN and 6 PCS patients had positive clinical HIT. Mean time to testing was 4.7 days for VN, 7.0 days for PCS. $\mathrm{VN}$ mean ipsilesional $\mathrm{HC}$ and $\mathrm{AC}$ first saccade amplitude was larger, peak-velocities faster and onset latencies earlier compared to PCS $(\mathrm{p}<0.05)$. No significant difference between VN and PCS in first saccade characteristics was found in PC. Ipsilesional first saccade amplitude, peak-velocity and duration were significantly different between PCS and controls for all canals $(\mathrm{p}<0.05)$. A gain $<0.68$ and first saccade amplitudes $>2.2^{\circ}$ separated VN from PCS with sensitivities of $95.5 \%$ and $86.4 \%$ and specificities of $72.7 \%$ and $63.6 \%$. First saccade amplitude of $>0.91^{\circ}$ identified PCS from controls with sensitivity of $68.2 \%$ and specificity of $70 \%$. Abnormality rates for AC cVEMP, BC oVEMP and SVH were 42.9\%, 50\% and $91 \%$ for $\mathrm{VN}$ and $38.1 \%$, 9\% $72 \%$ for PCS.
Conclusion v-HIT gain and catch-up saccade metrics are useful separators of VN from PCS. Detailed saccade analysis complements existing vestibular tests.

\section{SYNDROME OF TRANSIENT HEADACHE AND NEUROLOGIC DEFICITS WITH CEREBRAL FLUID LYMPHOCYTOSIS (HANDL) AS A MIMIC FOR TRANSIENT ISCHAEMIC ATTACK (TIA)}

Jasmine F Ashhurst* ${ }^{*}$ Rami Haddad. Orange Hospital, Orange, NSW, Australia

\subsection{6/jnnp-2019-anzan.73}

Introduction $\mathrm{HaNDL}$ is a rare neurological disorder of unknown aetiology that is characterised by headache, neurological deficit and pleocytosis in the cerebrospinal fluid (CSF). It is a benign condition that has spontaneous resolution of symptoms within months.

A 50 year old female presented to Emergency with an acute focal neurological deficit of right sided weakness, dysphasia and dysarthria lasting less than one hour. Over the preceding months she had a new onset of headache. She was worked up for a likely diagnosis of TIA.

Methods Case report.

Results Initial CT Brain(angiography) showed no stroke or other identifiable cause of symptoms.

MRI brain showed excessive nonspecific T2 hyperintensities, requiring further investigation for possible vasculitis. MR angiography was normal and there was no evidence of stroke.

Viral PCR's were negative.

Lumbar puncture (LP) showed pleocytosis (leucocytes $309 \times 10 \mathrm{E} 6 / \mathrm{L}$ ), raised intracranial pressure and high protein, raising suspicion for HaNDL. This normalised on subsequent outpatient LP, along with symptoms.

Conclusions

A diagnosis of HaNDL is made as a diagnosis of exclusion, though should be considered as a differential diagnosis for various presentations in which transient acute focal neurology is a presenting complaint.

Due to relatively few reported cases of HaNDL, it is possible that HaNDL is being underdiagnosed due to variability in patient presentation and lack of understanding of the syndrome.

As in this case, when a patient presents with transient acute focal neurology in the absence of headache as a prominent presenting symptom, it is reasonable to consider HaNDL as a differential diagnosis.

\section{CLINICAL, INVESTIGATIONAL AND TREATMENT FACTORS DO NOT DETERMINE PROGNOSIS OF PATIENTS WITH INFLAMMATORY NEUROPATHIES}

\footnotetext{
${ }^{2,1}$ Mahima Kapoor* ${ }^{2,3}$ Aisling Carr, ${ }^{3}$ Michael P Lunn, ${ }^{4}$ Mary M Reilly. ${ }^{1}$ Central Clinical School, Monash University, Melbourne, VIC, Australia; ${ }^{2}$ MRC Centre for Neuromuscular Diseases, UCL Institute of Neurology, University College London, London, UK; ${ }^{3}$ National Hospital for Neurology and Neurosurgery, London, UK; ${ }^{4} M R C$ Centre for Neuromuscular Diseases, UCL Institute of Neurology, University College London, London, UK
}

\subsection{6/jnnp-2019-anzan.74}

Introduction Identifying patients who need long-term immunoglobulin (IVIg) treatment in patient with inflammatory 